\title{
EDITORIALE
}

\section{Italian Journal of Medicine online su ScienceDirect}

\section{Italian Journal of Medicine now online in ScienceDirect}

\section{Cari soci, lettori e autori}

a partire da questo numero, Italian Journal of Medicine, Organo Ufficiale della Federazione delle Associazioni dei Dirigenti Ospedalieri Internisti (FADOI), è pubblicato anche online sulla piattaforma elettronica ScienceDirect.

Siamo molto soddisfatti di questo risultato, ulteriore segnale della crescita della nostra Società e frutto della fattiva collaborazione di tutti voi e dell'aiuto dell'Editore Elsevier.

ScienceDirect è la piattaforma elettronica più evoluta nel campo delle pubblicazioni medico-scientifiche. Vi sono pubblicate più di 2.500 riviste, tra cui The Lancet e la sua numerosa famiglia di periodici specialistici, Cell, e molte altre pubblicazioni prestigiose del panorama medico-scientifico internazionale. I visitatori della piattaforma in tutto il mondo sono oltre 11 milioni ogni anno, con 1 milione di autori pubblicati.

Siamo convinti che Italian Journal of Medicine beneficierà moltissimo della pubblicazione online su ScienceDirect:

- la visibilità della testata aumenterà notevolmente, e così i livelli di lettura degli articoli e la possibilità di citazione degli stessi;

- la pubblicazione degli articoli online sarà anticipata rispetto a quella sulla rivista cartacea e avverrà a breve distanza dall'accettazione dei manoscritti per la pubblicazione;

- gli articoli, fin dalla loro pubblicazione anticipata, saranno dotati di un Digital Object Identifier (DOI), che ne consente la citazione immediata;

- entro il 2009 ci proponiamo di implementare anche l'utilizzo dell'Elsevier Editorial System (EES) per l'invio elettronico dei manoscritti da parte degli autori e la gestione del processo di referaggio online;

Anche autori e lettori beneficeranno di questo passaggio alla pubblicazione online, grazie a:

- lettura degli articoli in formato html, con la possibilità di scaricare e stampare i file pdf degli articoli;

- un potente motore di ricerca (sull'intero testo e sugli abstract), con cui effettuare ricerche tra gli articoli pubblicati;
- attivazione di email alert per sapere quando un articolo è stato pubblicato online;

- possibilità per gli autori di tracciare il proprio manoscritto nella varie fasi di lavorazione;

- opzione Cited by, che consente di vedere tutti gli articoli pubblicati su ScienceDirect in cui un determinato manoscritto è stato citato;

- Save as Citation, alert che aggiorna ogni volta che uno specifico articolo è stato citato;

- possibilità di salvare ed editare ricerche già effettuate per riutilizzarle quando necessario.

E questi non sono che alcuni dei molti vantaggi della pubblicazione online.

In occasione di questo importante cambiamento, la grafica della testata è stata completamente rinnovata per avvicinarsi sempre più all'immagine delle riviste scientifiche internazionali.

I soci FADOI, oltre a continuare la ricevere la rivista in formato cartaceo, avranno anche la possibilità di accedere gratuitamente alla testata online tramite una homepage dedicata e personalizzata con il logo della società scientifica e utilizzando le chiavi di accesso che saranno fornite dell'Editore.

L'ufficio editoriale e la redazione di Elsevier a Milano continueranno ovviamente a essere a disposizione di autori e lettori in questo eccitante nuovo cammino.

Abbiamo lavorato tutti molto intensamente a questo progetto che vede finalmente la luce e vorremmo nuovamente ringraziare tutti per la fattiva collaborazione che ci ha consentito di raggiungere importanti risultati.

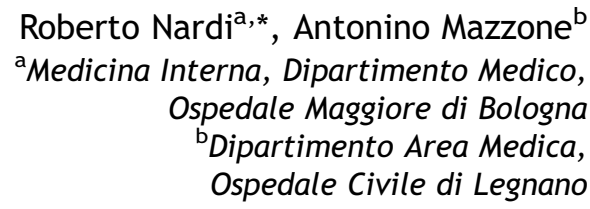

${ }^{*} E$-mail: r.nardi@ausl.bologna.it 